\title{
Haemodynamics and serial risk assessment in systemic sclerosis associated pulmonary arterial hypertension
}

\author{
Jason Weatherald (1) 1,2,3,4,5,20, Athénaïs Boucly ${ }^{1,2,3,20}$, David Launay ${ }^{6,7,8,9}$, \\ Vincent Cottin (10 ${ }^{10,11}$, Grégoire Prévot ${ }^{12}$, Delphine Bourlier ${ }^{13}$, Claire Dauphin ${ }^{14}$, \\ Ari Chaouat ${ }^{15,16,17}$, Laurent Savale ${ }^{1,2,3}$, Xavier Jaïs ${ }^{1,2,3}$, Mitja Jevnikar ${ }^{1,2,3}$, \\ Julie Traclet ${ }^{10,11}$, Pascal De Groote ${ }^{17}$, Gérald Simonneau ${ }^{1,2,3}$ \\ Eric Hachulla ${ }^{6,7,8,9}$, Luc Mouthon ${ }^{18,19}$, David Montani (1) ${ }^{1,2,3}$, \\ Marc Humbert ${ }^{1,2,3,21}$ and Olivier Sitbon (1) $1,2,3,21$
}

@ERSpublications

For newly diagnosed patients with systemic sclerosis-associated PAH, some haemodynamic variables, particularly the stroke volume index, and a multidimensional risk assessment were more useful during early follow-up than at baseline http://ow.ly/LUuj30lrvfW

Cite this article as: Weatherald J, Boucly A, Launay D, et al. Haemodynamics and serial risk assessment in systemic sclerosis associated pulmonary arterial hypertension. Eur Respir J 2018; 52: 1800678 [https://doi. org/10.1183/13993003.00678-2018].

ABSTRACT The prognostic importance of follow-up haemodynamics and the validity of multidimensional risk assessment are not well established for systemic sclerosis (SSc)-associated pulmonary arterial hypertension (PAH).

We assessed incident SSc-PAH patients to determine the association between clinical and haemodynamic variables at baseline and first follow-up right heart catheterisation (RHC) with transplantfree survival. RHC variables included cardiac index, stroke volume index (SVI), pulmonary arterial compliance and pulmonary vascular resistance. Risk assessment was performed according to the number of low-risk criteria: functional class I or II, 6-min walking distance (6MWD) $>440 \mathrm{~m}$, right atrial pressure $<8 \mathrm{mmHg}$ and cardiac index $\geqslant 2.5 \mathrm{~L} \cdot \mathrm{min}^{-1} \cdot \mathrm{m}^{-2}$.

Transplant-free survival from diagnosis $(n=513)$ was $87 \%, 55 \%$ and $35 \%$ at 1,3 and 5 years, respectively. At baseline, 6MWD was the only independent predictor. A follow-up RHC was available for 353 patients (median interval 4.6 months, interquartile range 3.9-6.4 months). The 6MWD, functional class, cardiac index, SVI, pulmonary arterial compliance and pulmonary vascular resistance were independently associated with transplant-free survival at follow-up, with SVI performing better than other haemodynamic variables. 1-year outcomes were better with increasing number of low-risk criteria at baseline (area under the curve (AUC) 0.63, 95\% CI 0.56-0.69) and at first follow-up (AUC 0.71, 95\% CI 0.64-0.78).

Follow-up haemodynamics and multidimensional risk assessment had greater prognostic significance than at baseline in SSc-PAH. 
Affiliations: 'Université Paris-Sud, Faculté de Médecine, Université Paris-Saclay, Le Kremlin-Bicêtre, France. ${ }^{2}$ APHP, Hôpital Bicêtre, Service de Pneumologie, Le Kremlin-Bicêtre, France. ${ }^{3}$ INSERM UMR_S 999, Hôpital Marie Lannelongue, Le Plessis Robinson, France. ${ }^{4}$ Dept of Medicine, Division of Respirology, University of Calgary, Calgary, AB, Canada. ${ }^{5} \mathrm{Libin}$ Cardiovascular Institute of Alberta, University of Calgary, Calgary, AB, Canada. 'Université Lille, U995 - LIRIC - Lille Inflammation Research International Center, Lille, France. ${ }^{7}$ INSERM, U995, Lille, France. ${ }^{8} \mathrm{CHU}$ Lille, Département de Médecine Interne et Immunologie Clinique, Lille, France. ${ }^{9}$ Centre de Référence des Maladies Autoimmunes et Systémiques Rares du Nord et Nord-Ouest de France (CeRAINO), Lille, France. ${ }^{10}$ Hospices Civils de Lyon, Dept of Respiratory Medicine, Louis Pradel Hospital, Lyon, France. ${ }^{11}$ University Claude Bernard Lyon 1, University of Lyon, UMR 754, Lyon, France. ${ }^{12} \mathrm{CHU}$ de Toulouse, Hôpital Larrey, Service de Pneumologie, Toulouse, France. ${ }^{13}$ Université de Bordeaux, CHU de Bordeaux, Hôpital du Haut Lévêque, Service de Maladies Respiratoires, Pessac, France. ${ }^{14}$ University of Auvergne, CHU Clermont-Ferrand, Cardiology Department, Clermont-Ferrand, France. ${ }^{15} \mathrm{CHU}$ Nancy, Pôle des Spécialités Médicales, Département de Pneumologie, Vandoeuvre-lès-Nancy, France. ${ }^{16}$ Université de Lorraine, INGRES, EA 7298, Vandoeuvre-lès-Nancy, France. ${ }^{17} \mathrm{CHU}$ de Lille, Hôpital Cardiologique de Lille, Centre de Compétences de l'Hypertension Pulmonaire, Lille, France. ${ }^{18}$ APHP, Hôpital Cochin, Service de Médecine Interne, Centre de Référence des Maladies Auto-immunes Systémiques Rares Ile de France, Paris, France. ${ }^{19}$ Université Paris Descartes, Paris, France. ${ }^{20}$ Both authors contributed equally. ${ }^{21}$ Both authors contributed equally.

Correspondence: Olivier Sitbon, Service de Pneumologie et Soins Intensifs, CHU de Bicêtre, 78 rue du General Leclerc, F-94275, Le Kremlin-Bicêtre, France. E-mail: olivier.sitbondaphp.fr

\section{Introduction}

Pulmonary arterial hypertension (PAH) develops in $\sim 10 \%$ of individuals with systemic sclerosis (SSc) [1] and, when present, is one of the most frequent causes of death in these patients [2]. The diagnosis of PAH requires haemodynamic measurements during right heart catheterisation (RHC), with a mean pulmonary artery pressure $(\mathrm{mPAP}) \geqslant 25 \mathrm{mmHg}$ and pulmonary artery wedge pressure $\leqslant 15 \mathrm{mmHg}$ [3].

While essential for the diagnosis of PAH, haemodynamic variables such as the right atrial pressure (RAP), cardiac index and stroke volume provide important information about right ventricular (RV) function, and predict survival in SSc-PAH [4-6]. Several observational studies, predominantly of idiopathic PAH (IPAH) patients, have noted the importance of clinical variables, such as the 6-min walking distance (6MWD), New York Heart Association functional class (NYHA-FC) and haemodynamic variables achieved during early follow-up as they reflect response to initial PAH therapy [7-10]. The long-term prognostic significance of early follow-up clinical and haemodynamic variables after initial treatment has not been specifically studied in the SSc-PAH population.

Periodic risk assessment using a multidimensional approach is recommended by recent European Society of Cardiology (ESC) and European Respiratory Society (ERS) guidelines, with thresholds for a "low-risk" patient defined by several clinical, exercise, imaging-derived and haemodynamic parameters [3]. A study from the French PAH registry demonstrated that the number of low-risk criteria present was a simple and valid method of assessing risk in IPAH and heritable and drug-induced PAH [11]. However, the validity of this approach to risk assessment has not been studied specifically in SSc-PAH.

The main objective of this study was to evaluate the prognostic value of haemodynamic variables at baseline and after initial treatment in newly diagnosed SSc-PAH patients. A secondary objective was to determine whether the number of low-risk criteria at baseline and first follow-up RHC was associated with transplant-free survival in SSc-PAH.

\section{Methods}

This study complied with the Declaration of Helsinki. Although French law does not require ethics committee approval or informed consent for retrospective data collection, the data collected in the French Pulmonary Arterial Hypertension Network registry were anonymised and complied with the requirements of the Commission Nationale Informatique et Liberté (CNIL). CNIL, the organisation dedicated to privacy, information technology and civil rights in France, approved the methods used to collect and analyse data on May 24, 2003 (approval number 842063).

\section{Study population}

Incident, treatment-naïve patients in the French Pulmonary Arterial Hypertension Network registry with SSc-PAH were prospectively enrolled between January 2006 and March 2017. Patients were eligible for inclusion if they had a diagnosis of SSc, were $\geqslant 18$ years of age, and had newly diagnosed group 1 precapillary $\mathrm{PAH}$ on $\mathrm{RHC}$, defined as resting $\mathrm{mPAP} \geqslant 25 \mathrm{mmHg}$, pulmonary artery wedge pressure $\leqslant 15 \mathrm{mmHg}$ and pulmonary vascular resistance (PVR) $>3$ Wood units [3]. The diagnosis of SSc was made according to current criteria by the treating physician according to American College of Rheumatology/ European League Against Rheumatism criteria [12]. Patients with a recorded history of severe interstitial 
lung disease on computed tomography in the registry, those coded as having group 2 (secondary to left heart disease), group 3 (due to lung disease or chronic hypoxaemia), group 4 (chronic thromboembolic pulmonary hypertension) or with missing baseline haemodynamic variables were excluded [3]. Classification of patients as having group $1 \mathrm{PAH}$ was at the discretion of clinicians at each site.

\section{Measurements}

Variables were assessed at baseline and at the time of the first follow-up RHC within 12 months of diagnosis. We recorded standard haemodynamic variables such as RAP, mPAP, cardiac output, cardiac index, PVR and mixed venous oxygen saturation $\left(\mathrm{S}_{\mathrm{vO}}\right)$, and calculated variables: stroke volume index (SVI) from the cardiac index divided by heart rate, and pulmonary arterial compliance (PCa), calculated by stroke volume divided by pulse pressure (the difference between systolic and diastolic pulmonary arterial pressure). The initial treatment strategy was defined according to number of PAH medications prescribed within 4 months of the initial RHC. Low-risk criteria were defined as NYHA-FC I or II, $6 \mathrm{MWD}>440 \mathrm{~m}, \mathrm{RAP}<8 \mathrm{mmHg}$ and cardiac index $\geqslant 2.5 \mathrm{~L} \cdot \mathrm{min}^{-1} \cdot \mathrm{m}^{-2}$ [3]. The primary outcome was death or lung transplantation. Transplant-free survival time was defined from the date of initial RHC until the occurrence of a primary outcome event for the baseline analysis and from the date of first follow-up RHC until the occurrence of a primary outcome event for the follow-up analysis. Patients without an outcome event were censored at the time of last clinical contact.

\section{Statistical analysis}

Continuous variables are expressed as mean \pm SD for normally distributed variables or median (interquartile range (IQR)) for non-normally distributed variables. Normality was assessed using the KolmogorovSmirnov test. Categorical variables are presented as $\mathrm{n}(\%)$. Changes from baseline to follow-up were assessed using a paired t-test, Wilcoxon signed-rank test or Chi-squared test where appropriate. All comparisons were two-sided, with a $\mathrm{p}$-value $<0.05$ considered significant. Cox proportional-hazards regression and Kaplan-Meier analysis were used to assess the association between variables at baseline, at first follow-up RHC and the number of low-risk criteria with transplant-free survival. The proportional-hazards assumption was tested using log-minus-log plots and collinearity of haemodynamic variables was assessed using linear regression or Spearman's rank correlation. Variables with a p-value $\leqslant 0.1$ in the univariable analysis were eligible for entry into the multivariable models only if they were not highly correlated (absolute value of Pearson's $r$ or Spearman's $\rho<0.6$ ) with other continuous variables and if $<25 \%$ of individuals had missing values for that variable. Case-wise deletion was used for multivariable modelling with no imputation for missing values. Statistical analyses were performed using STATA version 13.1 for Mac (College Station, TX, USA).

\section{Role of the funding source}

The funding bodies supporting this work had no role in the study design, data collection, analysis, interpretation of the data or writing of the manuscript.

\section{Results}

Study population and treatment strategies

Of 573 potentially eligible patients in the registry between 2006 and 2017, 513 were included in the baseline analysis (figure 1). Patient characteristics are shown in table 1. The majority were female (78.2\%) and had limited systemic sclerosis (69.2\%). Most patients were in NYHA-FC III (59.6\%) and 13.4\% were

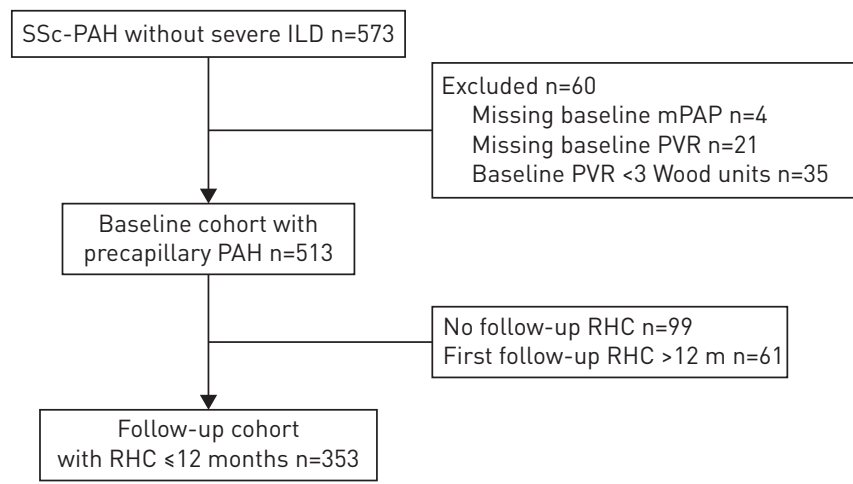

FIGURE 1 Study inclusion diagram. SSc-PAH: systemic sclerosis-associated pulmonary arterial hypertension; ILD: interstitial lung disease; mPAP: mean pulmonary arterial pressure; PVR: pulmonary vascular resistance; $\mathrm{RHC}$ : right heart catheterisation. 
in NYHA-FC IV. The most frequent initial treatment strategy was monotherapy (56.1\%) followed by initial combination therapy with two PAH therapies (31.4\%). Six (1.2\%) patients received initial triple combination therapy. Death occurred in 256 (49.9\%) patients during follow-up and 12 (2.3\%) received lung transplantation. Total follow-up from diagnosis was 1331.88 person-years with a median (IQR) follow-up time of $2.09(0.96-3.69)$ years. Median transplant-free survival from the time of diagnosis was 3.42 years (41 months) in the total population, and $1-, 3$ - and 5 -year rates were $87 \%$, $55 \%$ and $35 \%$, respectively (online supplementary figure S1).

\section{TABLE 1 Baseline characteristics}

Subjects

\begin{tabular}{|c|c|c|}
\hline Age years & 513 & $67.8(58.7-74.8)$ \\
\hline Female & 513 & 401 (78.2) \\
\hline BMI $\mathrm{kg} \cdot \mathrm{m}^{-2}$ & 504 & $24.2(21.2-27.7)$ \\
\hline BSA m ${ }^{2}$ & 506 & $1.67(1.53-1.82)$ \\
\hline \multicolumn{3}{|l|}{ Scleroderma subtype } \\
\hline Limited cutaneous & 513 & 355 (69.2) \\
\hline Diffuse cutaneous & 513 & $158(30.8)$ \\
\hline \multicolumn{3}{|l|}{ Auto-antibodies } \\
\hline Antinuclear antibody & 310 & $290(93.6)$ \\
\hline Anticentromere & 99 & $54(54.6)$ \\
\hline Anti-Scl 70 & 95 & $20(21.1)$ \\
\hline Anti-Ro & 59 & 7 (11.9) \\
\hline Anti-RNP & 78 & $4(5.13)$ \\
\hline Anti-SSA & 91 & $21(23)$ \\
\hline Anti-SSB & 85 & $4(4.7)$ \\
\hline \multicolumn{3}{|l|}{ Pulmonary function } \\
\hline FVC \% pred & 387 & $85(68-102)$ \\
\hline FEV $1 \%$ pred & 403 & $83(68-98)$ \\
\hline TLC $\%$ pred & 367 & $83(71-96)$ \\
\hline$D\left\llcorner c o / V_{A} \%\right.$ pred & 330 & $49(40-61)$ \\
\hline $\mathrm{PaO}_{2} \mathrm{mmHg}$ & 285 & $64(55-75)$ \\
\hline $\mathrm{PaCO}_{2} \mathrm{mmHg}$ & 279 & $33(30-37)$ \\
\hline Haemoglobin $\mathrm{g} \cdot \mathrm{L}^{-1}$ & 190 & $134(121-148)$ \\
\hline Creatinine $\mu \mathrm{mol} \cdot \mathrm{L}^{-1}$ & 192 & $82(71-104)$ \\
\hline NT-proBNP ng $\cdot \mathrm{L}^{-1}$ & 86 & 1143.5 (362-2873) \\
\hline$B N P \mathrm{ng} \cdot \mathrm{L}^{-1}$ & 295 & $320(87-693)$ \\
\hline $6 \mathrm{MWD}^{\#} \mathrm{~m}$ & 435 & $285(168-364)$ \\
\hline NYHA-FC & 500 & \\
\hline I & & $8(1.6)$ \\
\hline II & & $127(25.3)$ \\
\hline III & & $298(59.6)$ \\
\hline IV & & $67(13.4)$ \\
\hline \multicolumn{3}{|l|}{ Haemodynamics } \\
\hline $\mathrm{RAP} \mathrm{mmHg}$ & 485 & $6(4-10)$ \\
\hline sPAP mmHg & 501 & $66(54-77)$ \\
\hline $\mathrm{dPAP} \mathrm{mmHg}$ & 498 & $24(20-28)$ \\
\hline $\mathrm{mPAP} \mathrm{mmHg}$ & 513 & $40(34-47)$ \\
\hline PAWP $\mathrm{mmHg}$ & 506 & $8.4 \pm 3.4$ \\
\hline Cardiac output L. $\min ^{-1}$ & 513 & $4.12(3.33-5.07)$ \\
\hline Cardiac index $\mathrm{L} \cdot \mathrm{min}^{-1} \cdot \mathrm{m}^{-2}$ & 506 & $2.52(2.04-2.97)$ \\
\hline $\mathrm{SvO}_{2} \%$ & 287 & $65(59.6-71)$ \\
\hline $\mathrm{SV} \mathrm{mL}$ & 399 & $53(41-66)$ \\
\hline $\mathrm{SVI} \mathrm{mL} \cdot \mathrm{m}^{-2}$ & 394 & $32(25-39)$ \\
\hline PVR Wood units & 506 & $7.5(5.0-11.0)$ \\
\hline $\mathrm{PCa} \mathrm{mL} \cdot \mathrm{mmHg}^{-1}$ & 393 & $1.33(0.87-1.84)$ \\
\hline Heart rate bpm & 399 & $81(70-91)$ \\
\hline PAH treatment within first 4 months & 513 & \\
\hline No PAH therapy & & $56(10.9)$ \\
\hline Monotherapy & & $288(56.1)$ \\
\hline ERA & & 223 \\
\hline PDE5i or sGCS & & 60 \\
\hline PCA & & 5 \\
\hline
\end{tabular}


TABLE 1 Continued

Subjects

\begin{tabular}{lc} 
Dual therapy & $161(31.4)$ \\
ERA+PDE5i & 139 \\
ERA+PCA & 13 \\
PDE5i+PCA & 9 \\
Triple therapy (ERA+PDE5i+PCA) & $6(1.2)$ \\
CCB only & $2(0.4)$ \\
Diuretic & $299(58.3)$ \\
Anticoagulant & $226(44.1)$ \\
\hline
\end{tabular}

Data are presented as $\mathrm{n}$, median (interquartile range), mean $\pm \mathrm{SD}$, or $\mathrm{n}(\%)$. BMI: body mass index; BSA: body surface area; FVC: forced vital capacity; FEV1: forced expiratory volume in $1 \mathrm{~s}$; TLC: total lung capacity; $D\left\llcorner C o / V_{A}\right.$ : diffusing capacity of the lung for carbon monoxide adjusted for alveolar volume; $\mathrm{PaO}_{2}$ : arterial oxygen tension; $\mathrm{PaCO}_{2}$ : arterial carbon dioxide tension; NT-proBNP: N-terminal pro-brain natriuretic peptide; BNP: brain natriuretic peptide; 6MWD: 6-min walking distance; NYHA-FC: New York Heart Association functional class; RAP: right atrial pressure; sPAP: systolic pulmonary arterial pressure; dPAP: diastolic pulmonary arterial pressure; mPAP: mean pulmonary arterial pressure; PAWP: pulmonary artery wedge pressure; $\mathrm{SvO}_{2}$ : mixed venous oxygen saturation; SV: stroke volume; SVI: stroke volume index; PVR: pulmonary vascular resistance; PCa: pulmonary arterial compliance; ERA: endothelin receptor antagonist; PDE5i: phosphodiesterase type-5 inhibitor; sGCS: soluble guanylate cyclase stimulator; PCA: prostacyclin analogue; CCB: calcium channel blocker. ${ }^{\#}$ : 42 additional patients could not perform the 6-min walk test due to clinical severity or instability.

Changes in clinical and haemodynamic variables with initial treatment

There were 353 patients with a follow-up RHC performed within a year of diagnosis (median interval 4.6 months, IQR 3.9-6.4 months). Changes in functional and haemodynamic variables from baseline to first follow-up are shown in table 2. There were significant improvements in the proportion of patients in NYHA-FC I or II $(24 \%$ versus $43.5 \%, \mathrm{p}<0.001)$, median 6MWD (300 m versus $318 \mathrm{~m}, \mathrm{p}<0.001)$ and most haemodynamic variables.

TABLE 2 Changes in exercise, functional capacity and haemodynamic variables at follow-up

\begin{tabular}{|c|c|c|c|c|}
\hline & Subjects $\mathrm{n}$ & Baseline & Follow-up & p-value \\
\hline NYHA-FC & & & & $<0.001$ \\
\hline I & & $4(1.2)$ & $10(2.9)$ & \\
\hline II & & 79 (22.8) & 142 (40.2) & \\
\hline III & & $217(62.5)$ & $173(49.4)$ & \\
\hline IV & & 47 (13.5) & $25(7.1)$ & \\
\hline 6MWD m & 310 & $300(180-366)$ & $318(220-390)$ & $<0.001$ \\
\hline RAP mmHg & 345 & $6(4-10)$ & $6(3-10)$ & 0.35 \\
\hline sPAP mmHg & 353 & $67(55-78)$ & $64(49-77)$ & $<0.001$ \\
\hline dPAP mmHg & 353 & $25(20-29)$ & $22(17-28)$ & $<0.001$ \\
\hline mPAP mmHg & 353 & $41(35-48)$ & $39(31-47)$ & $<0.001$ \\
\hline PAWP $\mathrm{mmHg}$ & 344 & $8(6-10)$ & $9(6-12)$ & $<0.001$ \\
\hline Cardiac output L.min ${ }^{-1}$ & 353 & $4.13(3.37-5.10)$ & $4.70(3.91-5.80)$ & $<0.001$ \\
\hline Cardiac index $L \cdot \min ^{-1} \cdot \mathrm{m}^{-2}$ & 349 & $2.49(2.04-2.92)$ & $2.86(2.35-3.39)$ & $<0.001$ \\
\hline $\mathrm{SvO}_{2} \%$ & 233 & $65(60-71)$ & $65(60-71)$ & 0.56 \\
\hline SV mL & 302 & $54(41-66)$ & $60(49-74)$ & $<0.001$ \\
\hline $\mathrm{SVI} \mathrm{mL} \cdot \mathrm{m}^{-2}$ & 301 & $32(26-38)$ & $36(30-43)$ & $<0.001$ \\
\hline PVR Wood units & 342 & $7.9(5.2-11.4)$ & $5.9(4.2-8.6)$ & $<0.001$ \\
\hline PCa $\mathrm{mL} \cdot \mathrm{mmHg}^{-1}$ & 302 & $1.36(0.87-1.85)$ & $1.55(1.11-2.28)$ & $<0.001$ \\
\hline Heart rate bpm & 302 & $80(70-91)$ & $80(70-90)$ & 0.46 \\
\hline
\end{tabular}

Data are presented as $\mathrm{n}(\%)$ or median (interquartile range), unless otherwise stated. $\mathrm{n}=353$. NYHA-FC: New York Heart Association functional class; 6MWD: 6-min walking distance; RAP: right atrial pressure; sPAP: systolic pulmonary arterial pressure; dPAP: diastolic pulmonary arterial pressure; mPAP: mean pulmonary arterial pressure; PAWP: pulmonary artery wedge pressure; $\mathrm{SvO}_{2}$ : mixed venous oxygen saturation; SV: stroke volume; SVI: stroke volume index; PVR: pulmonary vascular resistance; PCa: pulmonary arterial compliance. 
Baseline variables and transplant-free survival

The univariable associations between baseline characteristics and transplant-free survival are shown in table 3. In multivariable models that included age, BMI, 6MWD, NYHA-FC, RAP, mPAP and either cardiac index, SVI, PVR or PCa, the 6MWD was the only variable independently associated with

TABLE 3 Univariable analyses for baseline and follow-up factors and transplant-free survival

\begin{tabular}{|c|c|c|}
\hline & \multicolumn{2}{|c|}{ Baseline } \\
\hline & Hazard ratio $(95 \% \mathrm{CI})$ & p-value \\
\hline \multicolumn{3}{|l|}{ Baseline } \\
\hline Age (per year) & $1.02(1.01-1.03)$ & $<0.001$ \\
\hline Female sex & $0.84(0.62-1.13)$ & 0.256 \\
\hline BMI (per $\mathrm{kg} \cdot \mathrm{m}^{-2}$ ) & $0.97(0.94-0.99)$ & 0.01 \\
\hline Diffuse SSc & $1.04(0.79-1.37)$ & 0.77 \\
\hline Limited SSc & $0.98(0.74-1.29)$ & 0.86 \\
\hline FVC \% pred (per 10\%) & $0.94(0.88-0.997)$ & 0.04 \\
\hline FEV1 \% pred (per 10\%) & $0.95(0.89-1.01)$ & 0.125 \\
\hline TLC \% pred & $0.94(0.88-1.01)$ & 0.09 \\
\hline DLco/VA $\%$ pred (per 10\%) & $0.96(0.86-1.05)$ & 0.371 \\
\hline $\mathrm{PaO}_{2}($ per $10 \mathrm{mmHg})$ & $0.8(0.72-0.90)$ & $<0.001$ \\
\hline $\mathrm{PaCO}_{2}$ (per $10 \mathrm{mmHg}$ ) & $0.83(0.64-1.09)$ & 0.192 \\
\hline Haemoglobin & $1.002(1.00-1.003)$ & 0.007 \\
\hline Creatinine (per $10 \mu \mathrm{mol} \cdot \mathrm{L}^{-1}$ ) & $1.14(1.07-1.22)$ & $<0.001$ \\
\hline BNP (per $100 \mathrm{ng} \cdot \mathrm{L}^{-1}$ ) & $1.04(1.02-1.06)$ & 0.001 \\
\hline 6MWD (per $10 \mathrm{~m}$ ) & $0.97(0.96-0.98)$ & $<0.001$ \\
\hline \multicolumn{3}{|l|}{ NYHA-FC (versus NYHA III) } \\
\hline $\mathrm{I} / \mathrm{II}$ & $0.68(0.50-0.93)$ & 0.015 \\
\hline IV & $1.41(0.998-1.99)$ & 0.051 \\
\hline RAP & $1.02(0.996-1.05)$ & 0.096 \\
\hline mPAP (per 10 mmHg) & $1.15(1.02-1.31)$ & 0.026 \\
\hline PAWP & $0.98(0.94-1.02)$ & 0.283 \\
\hline Cardiac output & $0.81(0.74-0.90)$ & $<0.001$ \\
\hline Cardiac index & $0.74(0.61-0.89)$ & 0.001 \\
\hline $\mathrm{SvO}_{2}$ & $0.97(0.96-0.99)$ & 0.001 \\
\hline SV (per $10 \mathrm{~mL}$ ) & $0.87(0.80-0.94)$ & 0.001 \\
\hline SVI (per $10 \mathrm{~mL} \cdot \mathrm{m}^{-2}$ ) & $0.79(0.68-0.92)$ & 0.002 \\
\hline Heart rate & $1.00(0.99-1.01)$ & 0.377 \\
\hline PVR & $1.05(1.03-1.07)$ & $<0.001$ \\
\hline $\mathrm{PCa}$ & $0.63(0.51-0.77)$ & $<0.001$ \\
\hline \multicolumn{3}{|l|}{ Follow-up } \\
\hline 6MWD (per $10 \mathrm{~m})$ & $0.95(0.94-0.96)$ & $<0.001$ \\
\hline \multicolumn{3}{|l|}{ NYHA-FC (versus NYHA I/II) } \\
\hline III & $2.09(1.46-2.99)$ & $<0.001$ \\
\hline IV & $8.93(5.13-15.56)$ & $<0.001$ \\
\hline RAP (per mmHg) & $1.08(1.05-1.11)$ & $<0.001$ \\
\hline mPAP (per 10 mmHg) & $1.62(1.39-1.90)$ & $<0.001$ \\
\hline PAWP (per mmHg) & $0.99(0.95-1.03)$ & 0.648 \\
\hline Cardiac output (per L. $\min ^{-1}$ ) & $0.71(0.62-0.81)$ & $<0.001$ \\
\hline Cardiac index $\left(\right.$ per $\mathrm{L} \cdot \mathrm{min}^{-1} \cdot \mathrm{m}^{-2}$ ) & $0.50(0.39-0.63)$ & $<0.001$ \\
\hline $\mathrm{SvO}_{2}(\operatorname{per} 1 \%)$ & $0.98(0.96-0.99)$ & $<0.001$ \\
\hline SV (per $10 \mathrm{~mL}$ ) & $0.78(0.71-0.87)$ & $<0.001$ \\
\hline SVI (per $10 \mathrm{~mL} \cdot \mathrm{m}^{-2}$ ) & $0.57(0.47-0.70)$ & $<0.001$ \\
\hline Heart rate (per bpm) & $1.01(0.998-1.02)$ & 0.1 \\
\hline PVR (per Wood unit) & $1.2(1.14-1.25)$ & $<0.001$ \\
\hline $\mathrm{PCa}\left(\right.$ per $\left.\mathrm{mL} \cdot \mathrm{mmHg}^{-1}\right)$ & $0.57(0.45-0.72)$ & $<0.001$ \\
\hline
\end{tabular}

BMI: body mass index; SSc: systemic sclerosis; FVC: forced vital capacity; FEV1: forced expiratory volume in $1 \mathrm{~s}$; TLC: total lung capacity; DLCO/VA: diffusing capacity of the lung for carbon monoxide adjusted for alveolar volume; $\mathrm{PaO}_{2}$ : arterial oxygen tension; $\mathrm{PaCO}_{2}$ : arterial carbon dioxide tension; $\mathrm{BNP}$ : brain natriuretic peptide; 6MWD: 6-min walking distance; NYHA-FC: New York Heart Association functional class; RAP: right atrial pressure; mPAP: mean pulmonary arterial pressure; PAWP: pulmonary artery wedge pressure; $\mathrm{SvO}_{2}$ : mixed venous oxygen saturation; SV: stroke volume; SVI: stroke volume index; PVR: pulmonary vascular resistance; PCa: pulmonary arterial compliance. 


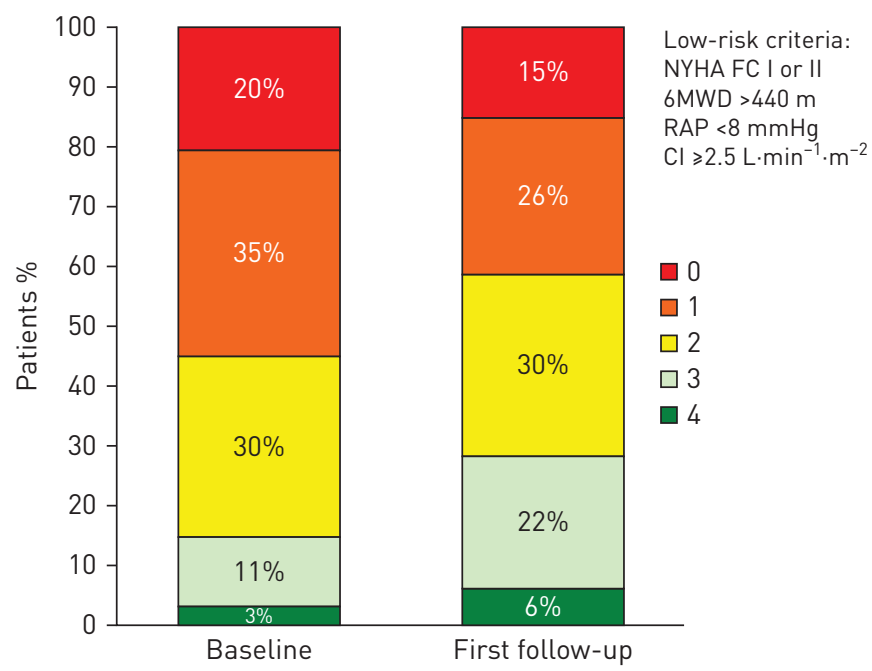

FIGURE 2 Number of low-risk criteria present at baseline and first follow-up right heart catheterisation. NYHA-FC: New York Heart Association functional class; 6MWD: 6-min walking distance; RAP: right atrial pressure; $\mathrm{Cl}$ : cardiac index.

transplant-free survival (adjusted hazard ratio (HR) 0.97 per $10 \mathrm{~m}$, 95\% CI 0.95-0.99 per $10 \mathrm{~m}$; p $<0.001$ ). Although several baseline haemodynamic variables were significant in the univariable analysis, none were independently associated, regardless of which variable (cardiac index, SVI, PCa or PVR) was entered, in the multivariable model.

\section{Risk assessment at baseline}

At baseline, the proportions of patients with $0,1,2,3$ or 4 low-risk criteria were $20 \%, 35 \%, 30 \%, 11 \%$ and $3 \%$, respectively (figure 2). There was a significantly lower risk of death or transplantation with increasing number of low-risk criteria (online supplementary table S1). Figure 3a shows transplant-free survival according to the number of low-risk criteria present at the time of PAH diagnosis. The ability of low-risk criteria at baseline to discriminate 1-year survival was modest with an area under the curve (AUC) of 0.63 (95\% CI 0.56-0.69) (online supplementary table S2).

\section{Follow-up variables and transplant-free survival}

After the first follow-up RHC, median (IQR) additional follow-up was $1.71(0.70-3.15)$ years and total observation time was 802.35 person-years. At the time of first follow-up RHC, the 6MWD, NYHA-FC and most haemodynamic variables were associated with transplant-free survival in univariable analysis (table 3 ). As cardiac index, SVI, PCa and PVR were highly correlated at follow-up (Spearman $\rho$ ranged from 0.62 to 0.87), separate multivariable models incorporating each haemodynamic variable with the other variables with $\mathrm{p} \leqslant 0.1$ in table 4 . In these multivariable models, the cardiac index, SVI, PVR and PCa at follow-up were independently associated with the primary outcome after adjusting for age, sex, 6MWD, NYHA-FC, RAP and mPAP. Because of a high degree of collinearity, mPAP was not included in the models with PVR $(\mathrm{r}=0.73, \mathrm{p}<0.001)$ or PCa $(\mathrm{r}=-0.67, \mathrm{p}<0.001)$. The SVI at first follow-up RHC had the lowest $-\log$ likelihood and Akaike's information criterion value. Figure 4 shows transplant-free survival according to quartiles for cardiac index, SVI, PVR and PCa at follow-up RHC (log-rank test $\mathrm{p}<0.001$ for all). Among patients who achieved or maintained cardiac index $\geqslant 2.5 \mathrm{~L} \cdot \mathrm{min}^{-1} \cdot \mathrm{m}^{-2}$ at the follow-up RHC, $28 \%$ had a SVI $<38 \mathrm{~mL} \cdot \mathrm{m}^{-2}[10]$ and these patients had significantly worse survival than those with an SVI $\geqslant 38 \mathrm{~mL} \cdot \mathrm{m}^{-2}$ (online supplementary figure $\mathrm{S} 2$ ).

\section{Risk assessment at follow-up}

At first follow-up RHC ( $\mathrm{n}=353)$, the proportions of patients with $0,1,2,3$ or 4 low-risk criteria were $15 \%$, $26 \%, 30 \%, 22 \%$ and $6 \%$, respectively (figure 2). Transplant-free survival was better with an increasing number of low-risk criteria present at first follow-up (figure 3b, online supplementary table S3) All low-risk criteria at follow-up were independently associated with outcomes (online supplementary table S4). Discriminating ability for 1-year outcome was reasonable (AUC 0.71, 95\% CI 0.64-0.78), such that having at least three low-risk criteria had $31.4 \%$ sensitivity and $92.7 \%$ specificity for surviving without lung transplantation in the subsequent year (online supplementary table S5). In addition, we performed an exploratory analysis of the 189 (54\%) patients with brain natriuretic peptide (BNP) or N-terminal 


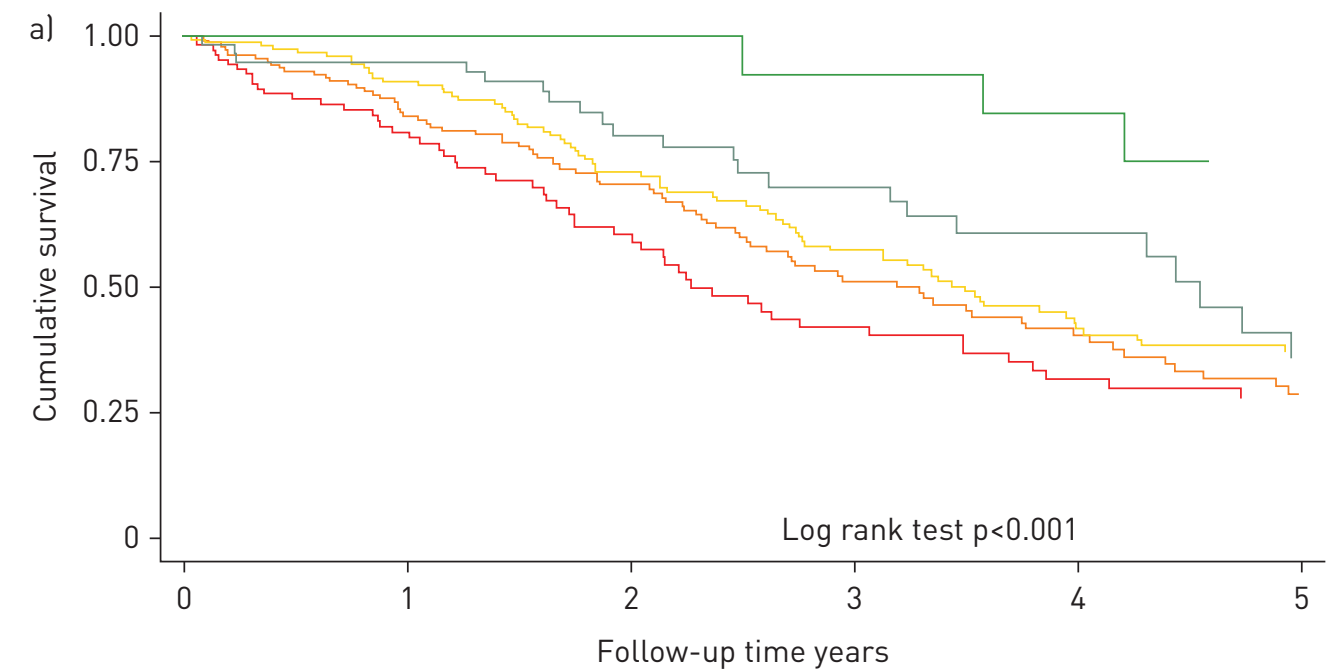

$\begin{array}{rlccccc}\text { Criteria } \mathrm{n} & \text { At risk } \mathrm{n} & & & & & \\ 0 & 105 & 71 & 40 & 26 & 31 & 14 \\ 1 & 177 & 118 & 86 & 49 & 38 & 18 \\ 2 & 156 & 123 & 90 & 62 & 16 & 29 \\ 3 & 58 & 52 & 34 & 24 & 10 & 7 \\ 4 & 17 & 17 & 15 & 12 & 10 & 5\end{array}$

Criteria: $-0-1-2-3-4$

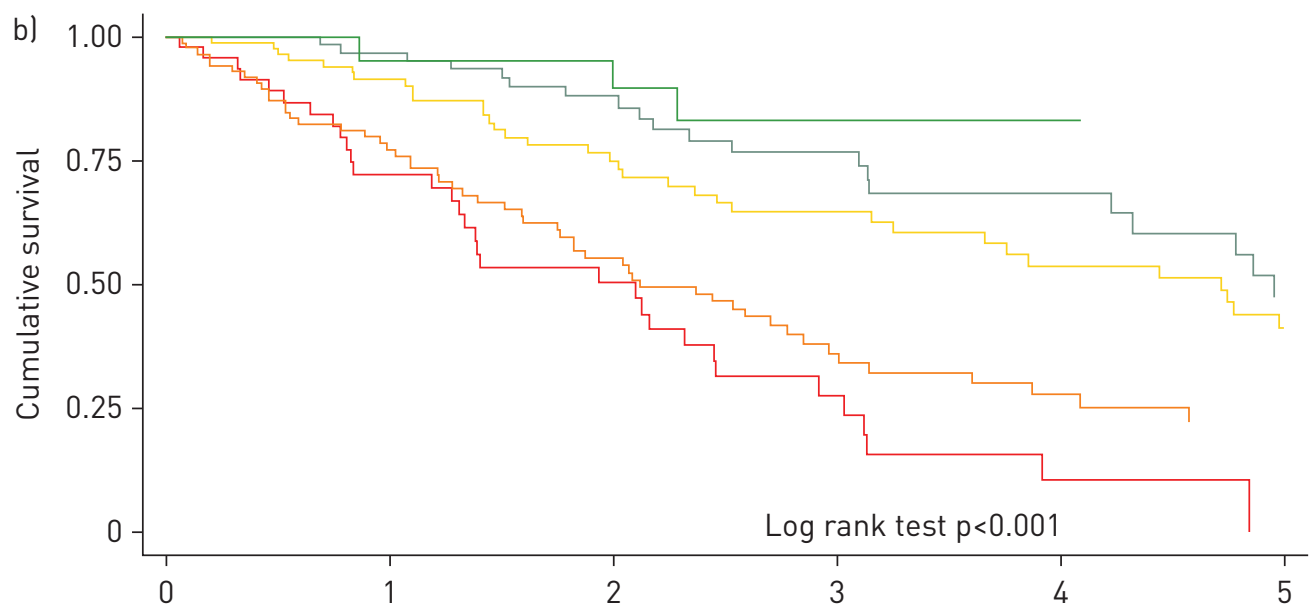

Criteria $\mathrm{n}$ At risk $\mathrm{n}$

$\begin{array}{ccccccc}0 & 53 & 28 & 16 & 7 & 2 & 0 \\ 1 & 94 & 60 & 38 & 19 & 10 & 8 \\ 2 & 105 & 68 & 45 & 34 & 23 & 14 \\ 3 & 79 & 60 & 40 & 30 & 17 & 11 \\ 4 & 22 & 20 & 15 & 12 & 9 & 7\end{array}$

FIGURE 3 Transplant-free survival according to the number of low-risk criteria present at a) baseline and b) first follow-up right heart catheterisation.

pro-BNP (NT-proBNP) at follow-up to determine whether achieving the low-risk thresholds for these biomarkers (NT-proBNP $<300 \mathrm{ng} \cdot \mathrm{L}^{-1}$ and $\mathrm{BNP}<50 \mathrm{ng} \cdot \mathrm{L}^{-1}$ ) was associated with transplant-free survival. Although having NT-proBNP/BNP low-risk criteria at follow-up was associated with better outcomes in univariable analysis (HR $0.31,95 \%$ CI $0.12-0.83 ; \mathrm{p}=0.02$ ), it was not independently associated when added to the other risk assessment criteria (online supplementary table S6). These results were similar whether or not the invasive haemodynamic criteria were included in the model (data not shown). 
TABLE 4 Multivariable analyses for clinical and haemodynamic variables at first follow-up right heart catheterisation

\begin{tabular}{|c|c|c|c|c|c|c|c|c|}
\hline & \multicolumn{2}{|c|}{ Model A\# } & \multicolumn{2}{|c|}{ Model B } & \multicolumn{2}{|c|}{ Model CI } & \multicolumn{2}{|c|}{ Model DT } \\
\hline & HR $(95 \%$ Cl) & $p$-value & HR $(95 \%$ CI) & p-value & HR $(95 \%$ Cl) & $p$-value & HR $(95 \%$ Cl) & p-value \\
\hline 6MWD (per $10 \mathrm{~m}$ ) & $0.96(0.94-0.97)$ & $<0.001$ & $0.96(0.94-0.97)$ & $<0.001$ & $0.96(0.94-0.97)$ & $<0.001$ & $0.96(0.94-0.97)$ & $<0.001$ \\
\hline \multicolumn{9}{|l|}{ NYHA-FC (versus I/II) } \\
\hline III & $1.07(0.71-1.63)$ & 0.33 & $1.07(0.68-1.67)$ & 0.775 & $1.1(0.70-1.71)$ & 0.68 & $1.19(0.78-1.81)$ & 0.41 \\
\hline IV & $3.43(1.62-7.28)$ & 0.001 & $3.43(1.49-7.92)$ & 0.004 & $3.55(1.52-8.25)$ & 0.003 & $3.26(1.53-6.96)$ & 0.002 \\
\hline RAP (per $1 \mathrm{mmHg}$ ) & $0.99(0.97-1.04)$ & 0.87 & $1(0.96-1.05)$ & 0.92 & $1.02(0.98-1.07)$ & 0.23 & $1.01(0.98-1.05)$ & 0.477 \\
\hline mPAP (per $10 \mathrm{mmHg}$ ) & $1.37(1.12-1.66)$ & 0.002 & $1.28(1.03-1.58)$ & 0.027 & & & & \\
\hline Cardiac index $\left(\right.$ per $\left.\mathrm{L} \cdot \mathrm{min}^{-1} \cdot \mathrm{m}^{-2}\right)$ & $0.59(0.45-0.78)$ & $<0.001$ & & & & & & \\
\hline SVI (per $10 \mathrm{~mL} \cdot \mathrm{m}^{-2}$ ) & & & $0.75(0.60-0.93)$ & 0.01 & & & & \\
\hline $\mathrm{PCa}\left(\right.$ per $\mathrm{mmHg} \cdot \mathrm{mL}^{-1}$ ) & & & & & $0.71(0.54-0.94)$ & 0.02 & & \\
\hline PVR (per Wood unit) & & & & & & & $1.13(1.07-1.19)$ & $<0.001$ \\
\hline Log likelihood & -535.592 & & -476.992 & & -480.783 & & -526.421 & \\
\hline Akaike Information Criteria & 1087.185 & & 969.985 & & 975.567 & & 1066.843 & \\
\hline
\end{tabular}

6MWD: 6-min walking distance; NYHA-FC: New York Heart Association functional class; RAP: right atrial pressure; mPAP: mean pulmonary arterial pressure; SVI: stroke volume index; PCa: pulmonary arterial compliance; PVR: pulmonary vascular resistance. ${ }^{\#}$ : all models adjusted for age and sex. Neither age nor sex were significant independent variables in any models; ": mPAP not included in model due to high correlation with PCa ( $r=-0.67)$ and PVR ( $r=0.73)$. 

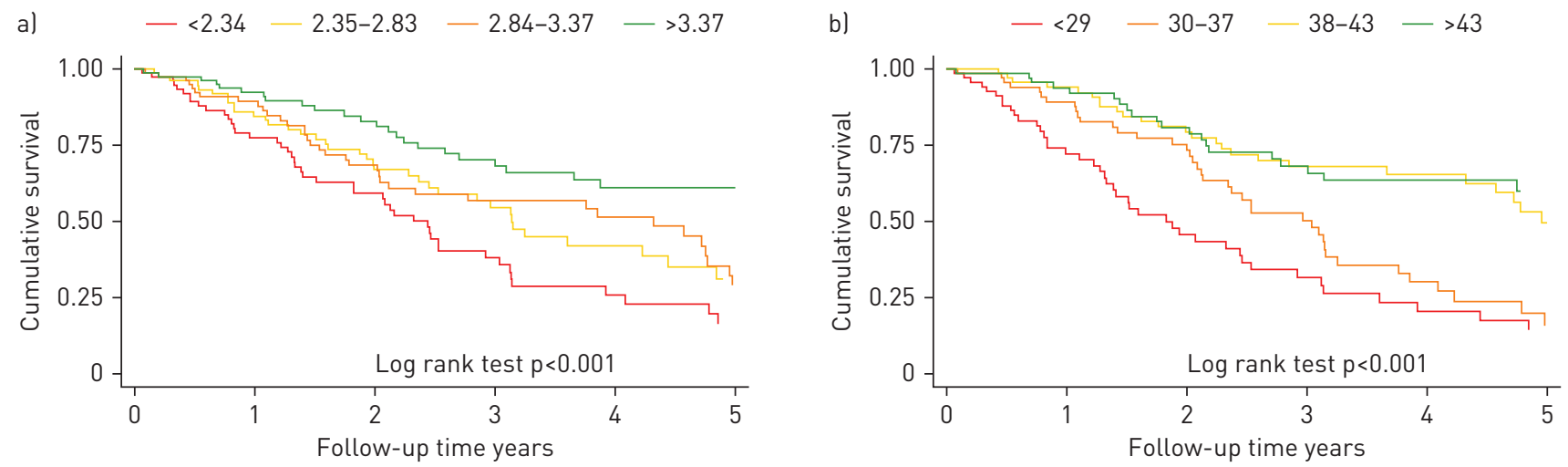

$\begin{array}{rllllll}\text { Cardiac index } & \text { At risk } n & & & & & \\ <2.34 & 86 & 51 & 32 & 17 & 9 & 5 \\ 2.35-2.83 & 87 & 58 & 37 & 24 & 12 & 7 \\ 2.84-3.37 & 88 & 58 & 36 & 27 & 18 & 9 \\ >3.37 & 88 & 66 & 47 & 34 & 22 & 19\end{array}$

volume index At risk $n$

$\begin{array}{rrrrrcc}<29 & 75 & 39 & 20 & 12 & 7 & 5 \\ 30-37 & 76 & 56 & 37 & 22 & 11 & 4 \\ 38-43 & 76 & 59 & 43 & 33 & 22 & 13 \\ >43 & 74 & 55 & 41 & 29 & 18 & 16\end{array}$
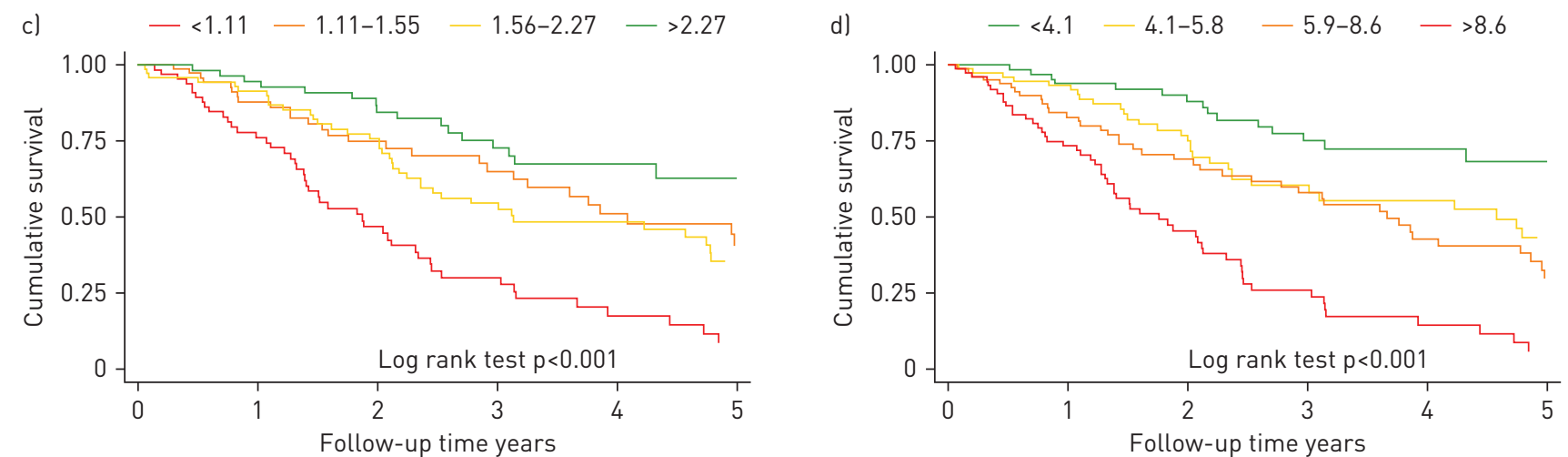

Pulmonary

Pulmonary

vascular

compliance At risk $n$

$\begin{array}{rrrrrrc}<1.11 & 76 & 44 & 23 & 13 & 6 & 3 \\ 1.11-1.55 & 76 & 54 & 33 & 25 & 17 & 11 \\ 1.56-2.27 & 75 & 59 & 47 & 29 & 19 & 12 \\ >2.27 & 75 & 53 & 39 & 29 & 16 & 12\end{array}$
resistance At risk $n$

$\begin{array}{rrrrrrc}<4.1 & 83 & 57 & 44 & 32 & 19 & 14 \\ 4.1-5.8 & 87 & 63 & 41 & 26 & 18 & 13 \\ 5.9-8.6 & 86 & 58 & 39 & 30 & 19 & 11 \\ >8.6 & 86 & 49 & 24 & 12 & 5 & 2\end{array}$

FIGURE 4 Transplant-free survival according to follow-up haemodynamic variable quartiles for a) cardiac index, b) stroke volume index, c) pulmonary arterial compliance and d) pulmonary vascular resistance.

\section{Discussion}

The main findings from this large, multicentre cohort study of incident SSc-PAH patients were: 1) baseline haemodynamic variables were not independent predictors of long-term transplant-free survival; 2) baseline risk assessment using the number of low-risk criteria, as suggested in the ESC/ERS guidelines, allowed modest discrimination of future risk; 3) follow-up haemodynamic variables within the first year of diagnosis were significantly and independently associated with transplant-free survival, with the SVI providing better explanation of the data than cardiac index, PVR or PCa; and 4) risk assessment using the number of low-risk criteria present at first follow-up RHC discriminated transplant-free survival better than at baseline. To our knowledge, this is the largest study to assess haemodynamics as prognostic factors in SSc-PAH and the first to compare the prognostic importance of haemodynamic variables achieved after initial treatment in this specific subgroup.

We confirmed our primary hypothesis that the SVI provides more useful clinical information than the cardiac index in patients with PAH, which was based upon our recent findings in patients with IPAH and drug-induced and heritable PAH [10]. In our SSc-PAH cohort, SVI at follow-up predicted transplant-free 
survival when adjusted for demographic, functional and RV loading variables (i.e. RAP and mPAP; table 4). Unadjusted transplant-free survival for patients in the highest two quartiles of SVI $\left(>37 \mathrm{~mL} \cdot \mathrm{m}^{-2}\right)$ at first follow-up were similar (figure 4), which is strikingly similar to the optimal cut-point of $38 \mathrm{~mL} \cdot \mathrm{m}^{-2}$ in patients with IPAH, heritable and drug-induced PAH [10], and which corresponds to the lower limit of normal for RV function [13]. Furthermore, we found that an important proportion of SSc-PAH patients who achieved a cardiac index goal of $\geqslant 2.5 \mathrm{~L} \cdot \mathrm{min}^{-1} \cdot \mathrm{m}^{-2}$ still had a low SVI $<38 \mathrm{~mL} \cdot \mathrm{m}^{-2}(28 \%)$ and a worse prognosis (online supplementary figure S 2), confirming that cardiac index can be falsely reassuring when the SVI is not also taken into account, and that SVI is likely a better haemodynamic indicator of RV function. While PCa reflects the pulsatile component of RV load and is calculated from the stroke volume [14], and PCa has been associated with prognosis in SSc-PAH patients $[5,9]$, our results suggest that the prognostic information provided by $\mathrm{PCa}$ is largely related to the stroke volume in SSc-PAH. Interestingly, in the study by CAMPO et al. [5], SVI and PCa at baseline were both associated with survival, but the model performance for each variable was quite similar, suggesting again that stroke volume is the main determinant of prognosis. Given the consistency of the association between SVI and prognosis across several PAH aetiologies, serial measures of SVI might be better in early phase or proof-of-concept clinical trials, to follow after treatment initiation or when assessing prognosis during follow-up.

RV function is a major determinant of survival in PAH and contractile function is worse in SSc-PAH than in IPAH patients for a similar degree of resistive or pulsatile afterload $[15,16]$. This discrepancy is likely related to intrinsic systolic dysfunction in SSc-PAH patients due to myocardial fibrosis and impaired sarcomere contractility as compared to IPAH patients [17]. Thus, markers of RV function are essential in assessing risk and therapeutic effect in SSc-PAH patients. While RHC remains mandatory for the diagnosis of PAH, non-invasive measures of RV function would be preferred during follow-up. The SVI and other measures of RV function can be obtained non-invasively with cardiac magnetic resonance imaging (MRI); however, it is not well established whether serial MRI measurements outperforms invasive prognostic variables in SSc-PAH. One study, which included connective tissue disease (CTD)-associated $\mathrm{PAH}$ patients, suggested that changes in RV ejection fraction distinguished survivors from non-survivors, whereas changes in cardiac index or PVR did not [18]. There is an increasing body of literature on the prognostic utility of MRI-derived indices of RV function in PAH, including the RV ejection fraction [19, 20], RV end-systolic volume [21], RV end-diastolic volume [22], SVI [22] and RV-pulmonary arterial coupling $[21,23]$. A recent MRI study by SwIFT et al. [21], which included 147 patients with CTD-PAH, found that $\mathrm{RV}$ end-systolic volume was prognostic in the overall cohort, but the ratio of RV elastance to arterial elastance $(E \mathrm{a})$, a load independent measure of RV-pulmonary arterial coupling, was independently associated with outcomes in the CTD-PAH group. In that study, the Ea was calculated from RHC and MRI measurements as (mPAP - PAWP (pulmonary arterial wedge pressure)) divided by SVI. Another study by VANDERPOOL et al. [23] found that entirely non-invasive MRI estimates of RV-pulmonary arterial coupling (calculated as stroke volume divided by end-systolic volume) were independently associated with survival [23]. Therefore, when repeated invasive haemodynamics are not possible, it may be useful to serially assess SVI, RV-pulmonary arterial coupling and other measures of RV function non-invasively for prognostication with MRI where it is readily available. Three-dimensional echocardiography-derived estimates of RV-pulmonary arterial coupling correlate well with MRI, which may be a future non-invasive prognostic tool [24]. In addition, the tricuspid annular plane systolic excursion derived from echocardiography reflects RV function [25] and has been associated with survival in SSc-PAH patients, albeit in a single small study [26].

PVR was independently predictive of transplant-free survival, although the model fit less well than those evaluating PCa and SVI. Others have also found that baseline PVR independently predicts survival in SSc-PAH [5, 27] although this is inconsistent [28]. Although RAP was not independently predictive in our multivariable models, it was associated in univariable analysis and this is a consistent finding in other studies of SSc-PAH $[5,29]$, which contrasts with the frequent observation that RAP is an independent prognostic factor in IPAH [10, 30-32]. Interestingly, having a low-risk RAP of $<8 \mathrm{mmHg}$ at follow-up was still independent of the other low-risk criteria (online supplementary table S4), and was an important variable in a previous meta-analysis [4], suggesting that a normal RAP is still an appropriate treatment goal for these patients.

Additionally, we confirmed the association between 6MWD and prognosis in SSc-PAH, even though the utility and validity of the 6MWD in SSc-PAH trials has been questioned due to other factors that affect exercise capacity in these patients, such as musculoskeletal limitations $[33,34]$ and because $6 \mathrm{MWD}$ changes correlate poorly with haemodynamic changes with treatment [35]. Nevertheless, in our study, the 6MWD was independently related to prognosis when measured at baseline or during follow-up. This suggests that the 6MWD still provides useful prognostic information, consistent with the meta-analysis by LEFĖvre et al. [4]. Another recent study by GADre et al. [36] derived a clinical score including the 6MWD, Borg dyspnoea score and oxygen saturation during the walk test, which was associated with 
haemodynamic severity and survival in SSc-PAH. Interestingly, while baseline 6MWD independently predicted long-term outcomes, haemodynamics and NYHA-FC at baseline did not. It may be that initial treatment decisions are more strongly influenced by the severity of haemodynamics or NYHA-FC rather than 6MWD in this population, with more severe haemodynamics or NYHA-FC III-IV invoking more aggressive combination treatment or use of parenteral therapies, which could mask or obviate the prognostic influence of baseline variables. The presence of NYHA-FC IV at follow-up was strongly related to mortality in our study, as would be expected for patients who had persistent severe functional impairment despite treatment.

When we assessed the number of low-risk criteria from the ESC/ERS guidelines [3] in our SSc-PAH population, we found that this simple tool is useful to assess risk as has been recently reported for IPAH, drug-induced and heritable PAH [11]. Similar to several previous studies, baseline assessment of risk did not discriminate short-term survival as well as risk assessment during follow-up, which emphasises the importance of response to treatment over baseline indices of disease severity [7, 11, 37, 38]. It is a sobering reminder of the dismal prognosis for SSc-PAH patients as only $28 \%$ of patients achieved three or four low-risk criteria after initial treatment and this "low-risk" group still had a $22 \%$ mortality rate at 3 years. Our results are similar to a recent study by HOEPER et al. [38] from the COMPERA registry, which included 347 patients with CTD-PAH at baseline and 213 at follow-up. They also demonstrated that few of the CTD-PAH patients achieved a low-risk profile after initial treatment, and these "low-risk" SSc-PAH patients had worse 5-year (55.5\%) survival compared to idiopathic, heritable and drug-induced PAH patients (72.4\%) [38]. In contrast to idiopathic, heritable and drug-induced PAH in the French registry [11] and idiopathic PAH patients from the COMPERA registry [39], we did not find that the NT-proBNP/BNP low-risk criteria added prognostic value in SSc-PAH patients. This may argue for the importance of invasive haemodynamic variables in SSc-PAH patients; however, a lack of association could be due to insufficient power as a result of missing data for NT-proBNP or BNP measurements at follow-up or the lack of data on renal function during follow-up, which could confound the association between these biomarkers and outcomes.

We must acknowledge some limitations to this study. It is important to recognise that the initial treatment strategy (monotherapy versus combination therapy) may explain the lack of independent association between baseline variables and outcomes, given that disease severity likely influenced treatment decisions, as has been proposed for other PAH subgroups [10]. The extent of missing data for certain variables that were significant in univariable analysis and which have been previously shown to be related to mortality in SSc-PAH, such as impaired renal function [5], $\mathrm{S}_{\mathrm{VO}_{2}}$ [40] and diffusion capacity of the lung for carbon monoxide [6] precluded their inclusion in multivariable analyses. As discussed earlier, the NT-proBNP and BNP analysis is limited by missing data for $46 \%$ of the follow-up cohort and lack of adjustment for renal function. Thus it remains unclear whether a non-invasive risk assessment using NYHA, 6MWD and NT-proBNP/BNP in SSc-PAH is accurate. Given a lack of echocardiographic data, we were unable to determine whether some patients had signs or features of left heart disease. Even though patients with PAWP $>15 \mathrm{mmHg}$ were excluded, the effect of occult left heart disease on haemodynamic variables cannot be ruled out. Since we limited our analysis to patients without any record of interstitial lung disease (ILD) in the registry, in order to assess a more homogenous population, it is not certain whether these results apply to those with mild ILD. However, another recent study of SSc patients with precapillary pulmonary hypertension from France and the United States suggested that outcomes in patients with a limited extent of ILD are similar to patients without ILD and PAH [41]. Lastly, although we were particularly interested in the value of haemodynamics during follow-up, the indications for RHC within the first year were not always clear and some patients did not undergo a follow-up RHC at all ( $\mathrm{n}=99$; figure 1). These patients who did not have any follow-up haemodynamic testing had similar baseline haemodynamics measurements and NYHA-FC as the rest of the cohort, but were significantly older (mean age 70.24 years versus 65.0 years, $\mathrm{p}<0.001$ ) and had lower baseline 6MWD ( $193 \pm 145 \mathrm{~m}$ versus $276 \pm 137 \mathrm{~m}, \mathrm{p}<0.001)$, thus, their exclusion could potentially have introduced a selection bias in our follow-up analysis.

\section{Conclusions}

In summary, we found that the haemodynamic variables during follow-up were independently associated with transplant-free survival in a large cohort of patients with SSc-PAH, whereas 6MWD was the only independent predictor at baseline. While traditional haemodynamic measures within the first year of treatment, including cardiac index and mPAP, were related to outcome, the SVI, PCa and PVR may represent more useful haemodynamic indicators of RV function and treatment targets. The number of low-risk criteria present at baseline and first follow-up RHC also predicted long-term outcomes, supporting the utility of this risk assessment tool in the SSc-PAH population. However, despite the fact that nearly a third of patients received combination therapy, only a minority of patients (28\%) achieved three or four low-risk criteria and long-term survival remains decidedly poor in this group of patients. 
Acknowledgements: We wish to acknowledge Laurence Rottat (Hôpital Bicêtre, Le Kremlin-Bicêtre, France) for her help in obtaining the data for this study and her hard work in managing data in the French PAH Registry.

Other contributors from the French PH network and registry (alphabetical order): Kais Ahmad (Lyon), Fabrice Bauer (Rouen), Emmanuel Bergot (Caen), Laurent Bertoletti (Saint Etienne), Arnaud Bourdin (Montpellier), Mathieu Canuet (Strasbourg), Céline Chabanne (Rennes), Bruno Degano (Besançon), Nicolas Favrolt (Dijon), Gilbert Habib (Marseille, La Timone), Delphine Horeau-Langlard (Nantes), Sylvie Leroy (Nice), Pascal Magro (Tours), Pierre Mauran (Reims), Jean-François Mornex (Lyon), Sylvain Palat (Limoges), Christophe Pison (Grenoble), Patrice Poubeau (La Réunion), Martine Reynaud-Gaubert (Marseille, Nord), Pascal Roblot (Poitiers), Olivier Sanchez (Paris-HEGP), Cécile Tromeur (Brest)

Author contributions: J. Weatherald and A. Boucly contributed equally to literature search, study design, data collection, analysis, figures, interpretation, writing. D. Launay: data collection, interpretation, writing. V. Cottin: data collection, interpretation, writing. G. Prévôt: data collection, interpretation, writing. D. Bourlier: data collection, interpretation, writing. C. Dauphin: data collection, interpretation, writing. A. Chaouat: data collection, interpretation, writing. L. Savale: data collection, interpretation, writing. X. Jaïs: data collection, interpretation, writing. M. Jevnikar: data collection, interpretation, writing. J. Traclet: data collection, interpretation, writing. P. De Groote: data collection, interpretation, writing. G. Simonneau: data collection, interpretation, writing. E. Hachulla: data collection, interpretation, writing. L. Mouthon: data collection, interpretation, writing. D. Montani: data collection, interpretation, writing. M. Humbert and O. Sitbon contributed equally to study design, data collection, analysis, interpretation, writing.

Conflict of interest: J. Weatherald reports grants from the European Respiratory Society and the Canadian Thoracic Society, during the conduct of the study; personal fees and non-financial support from Actelion Pharmaceuticals and Bayer, personal fees from Novartis, grants from Canadian Vascular Network, outside the submitted work. A. Boucly reports non-financial support from GSK, Bayer and MSD, and personal fees and non-financial support from Actelion, outside the submitted work. D. Launay reports grants from Pfizer, during the conduct of the study; and personal fees from Actelion, grants and personal fees from GSK, outside the submitted work. V. Cottin reports personal fees for consultancy, lecturing and travel to medical meetings from Actelion and Roche, personal fees for development of educational presentations, consultancy, lecturing and travel to medical meetings from Boehringer Ingelheim, personal fees for consultancy from Bayer and GSK, personal fees for adjudication committee work from Gilead, personal fees consultancy and travel to medical meetings from MSD, personal fees for consultancy and lecturing from Novartis and Sanofi, institutional grants from Boehringer Ingelheim and Roche, personal fees for chairing a DSMB from Promedior amd Celgene, and personal fees for consultancy and chairing a DSMB from Galapagos, outside the submitted work. G. Prévôt reports personal fees for consultancy, lecturing, development of educational presentations and travel to medical meetings from Actelion, Bayer, Boehringer Ingelheim, GSK and Roche, outside the submitted work. D. Bourlier reports personal fees from Novartis, outside the submitted work. C. Dauphin has nothing to disclose. A. Chaouat reports grants from Direction Générale de l'Offre de Soins (DGOS), during the conduct of the study, personal fees and non-financial support from Actelion Pharmaceuticals, Bayer and MSD, and non-financial support from GSK, outside the submitted work. L. Savale reports grants, personal fees and non-financial support from Actelion Pharmaceuticals, Bayer, GlaxoSmithKline and MSD and grants and personal fees from Pfizer, outside the submitted work. X. Jaïs reports personal fees and non-financial support from Actelion Pharmaceuticals, grants and personal fees from Bayer, and grants, personal fees and non-financial support from MSD and GSK, outside the submitted work. M. Jevnikar has nothing to disclose. J. Traclet reports personal fees from Actelion Pharmaceuticals, Boehringer Ingelheim and Roche, outside the submitted work. P. De Groote has nothing to disclose. G. Simonneau reports grants, personal fees and non-financial support from Actelion Pharmaceuticals, Bayer, GlaxoSmithKline and MSD and grants and personal fees from Pfizer, outside the submitted work. E. Hachulla has nothing to disclose. L. Mouthon has nothing to disclose. D. Montani reports grants and personal fees from Actelion and Bayer, and personal fees from BMS, GSK, MSD and Pfizer, outside the submitted work. M. Humbert has relationships with drug companies including Actelion, Bayer, GSK, Novartis and Pfizer. In addition to being investigator in trials involving these companies, relationships include consultancy service and membership of scientific advisory boards. O. Sitbon reports grants, personal fees and non-financial support from Actelion Pharmaceuticals, Bayer, GlaxoSmithKline and MSD, and grants and personal fees from Pfizer, outside the submitted work.

Support statement: This work was supported by a public grant overseen by the French National Research Agency (ANR) as part of the second Investissements d'Avenir programme (reference ANR-15-RHUS-0002). Jason Weatherald was the recipient of a joint European Respiratory Society/Canadian Thoracic Society Long-Term Research Fellowship (LTRF 2015-4780). Funding information for this article has been deposited with the Crossref Funder Registry.

\section{References}

1 Mukerjee D, St George D, Coleiro B, et al. Prevalence and outcome in systemic sclerosis associated pulmonary arterial hypertension: application of a registry approach. Ann Rheum Dis 2003; 62: 1088-1093.

2 Tyndall AJ, Bannert B, Vonk M, et al. Causes and risk factors for death in systemic sclerosis: a study from the EULAR Scleroderma Trials and Research (EUSTAR) database. Ann Rheum Dis 2010; 69: 1809-1815.

3 Galiè N, Humbert M, Vachiery J-L, et al. 2015 ESC/ERS Guidelines for the diagnosis and treatment of pulmonary hypertension: The Joint Task Force for the Diagnosis and Treatment of Pulmonary Hypertension of the European Society of Cardiology (ESC) and the European Respiratory Society (ERS). Eur Respir J 2015; 46: 903-975.

4 Lefèvre G, Dauchet L, Hachulla E, et al. Survival and prognostic factors in systemic sclerosis-associated pulmonary hypertension: a systematic review and meta-analysis. Arthritis Rheum 2013; 65: 2412-2423.

5 Campo A, Mathai SC, Le Pavec J, et al. Hemodynamic predictors of survival in scleroderma-related pulmonary arterial hypertension. Am J Respir Crit Care Med 2010; 182: 252-260.

6 Ramjug S, Hussain N, Hurdman J, et al. Idiopathic and systemic sclerosis-associated pulmonary arterial hypertension: a comparison of demographic, hemodynamic, and MRI characteristics and outcomes. Chest 2017; 152: $92-102$. 
7 Nickel N, Golpon H, Greer M, et al. The prognostic impact of follow-up assessments in patients with idiopathic pulmonary arterial hypertension. Eur Respir J 2012; 39: 589-596.

8 Galiè N, Jansa P, Pulido T, et al. SERAPHIN haemodynamic substudy: the effect of the dual endothelin receptor antagonist macitentan on haemodynamic parameters and NT-proBNP levels and their association with disease progression in patients with pulmonary arterial hypertension. Eur Heart J 2017; 38: 1147-1155.

9 Ghio S, D'Alto M, Badagliacca R, et al. Prognostic relevance of pulmonary arterial compliance after therapy initiation or escalation in patients with pulmonary arterial hypertension. Int J Cardiol 2017; 230: 53-58.

10 Weatherald J, Boucly A, Chemla D, et al. The prognostic value of follow-up hemodynamic variables after initial management in pulmonary arterial hypertension. Circulation 2018; 137: 693-704.

11 Boucly A, Weatherald J, Savale L, et al. Risk assessment, prognosis and guideline implementation in pulmonary arterial hypertension. Eur Respir J 2017; 50: 1700889

12 van den Hoogen F, Khanna D, Fransen J, et al. 2013 classification criteria for systemic sclerosis: an American College of Rheumatology/European League Against Rheumatism collaborative initiative. Ann Rheum Dis 2013; 72: $1747-1755$

13 Maceira AM, Prasad SK, Khan M, et al. Reference right ventricular systolic and diastolic function normalized to age, gender and body surface area from steady-state free precession cardiovascular magnetic resonance. Eur Heart J 2006; 27: 2879-2888.

14 Vonk Noordegraaf A, Westerhof BE, Westerhof N. The relationship between the right ventricle and its load in pulmonary hypertension. J Am Coll Cardiol 2017; 69: 236-243.

15 Tedford RJ, Mudd JO, Girgis RE, et al. Right ventricular dysfunction in systemic sclerosis-associated pulmonary arterial hypertension. Circ Heart Fail 2013; 6: 953-963.

16 Overbeek MJ, Lankhaar J-W, Westerhof $\mathrm{N}$, et al. Right ventricular contractility in systemic sclerosis-associated and idiopathic pulmonary arterial hypertension. Eur Respir J 2008; 31: 1160-1166.

17 Hsu S, Kokkonen-Simon KM, Kirk JA, et al. Right ventricular myofilament functional differences in humans with systemic sclerosis-associated versus idiopathic pulmonary arterial hypertension. Circulation 2018; 137: 2360-2370.

18 van de Veerdonk MC, Kind T, Marcus JT, et al. Progressive right ventricular dysfunction in patients with pulmonary arterial hypertension responding to therapy. J Am Coll Cardiol 2011; 58: 2511-2519.

19 Brewis MJ, Bellofiore A, Vanderpool RR, et al. Imaging right ventricular function to predict outcome in pulmonary arterial hypertension. Int J Cardiol 2016; 218: 206-211.

20 Baggen VJM, Leiner T, Post MC, et al. Cardiac magnetic resonance findings predicting mortality in patients with pulmonary arterial hypertension: a systematic review and meta-analysis. Eur Radiol 2016; 26: 3771-3780.

21 Swift AJ, Capener D, Johns C, et al. Magnetic resonance imaging in the prognostic evaluation of patients with pulmonary arterial hypertension. Am J Respir Crit Care Med 2017; 196: 228-239.

22 van Wolferen SA, Marcus JT, Boonstra A, et al. Prognostic value of right ventricular mass, volume, and function in idiopathic pulmonary arterial hypertension. Eur Heart J 2007; 28: 1250-1257.

23 Vanderpool RR, Pinsky MR, Naeije R, et al. RV-pulmonary arterial coupling predicts outcome in patients referred for pulmonary hypertension. Heart 2015; 101: 37-43.

24 Aubert R, Venner $\mathrm{C}$, Huttin $\mathrm{O}$, et al. Three-dimensional echocardiography for the assessment of right ventriculo-arterial coupling. J Am Soc Echocardiogr 2018; 31: 905-915.

25 Forfia PR, Fisher MR, Mathai SC, et al. Tricuspid annular displacement predicts survival in pulmonary hypertension. Am J Respir Crit Care Med 2006; 174: 1034-1041.

26 Mathai SC, Sibley CT, Forfia PR, et al. Tricuspid annular plane systolic excursion is a robust outcome measure in systemic sclerosis-associated pulmonary arterial hypertension. J Rheumatol 2011; 38: 2410-2418.

27 Mathai SC, Hummers LK, Champion HC, et al. Survival in pulmonary hypertension associated with the scleroderma spectrum of diseases: impact of interstitial lung disease. Arthritis Rheum 2009; 60: 569-577.

28 Williams MH, Das C, Handler CE, et al. Systemic sclerosis associated pulmonary hypertension: improved survival in the current era. Heart 2006; 92: 926-932.

29 Launay D, Sitbon O, Le Pavec J, et al. Long-term outcome of systemic sclerosis-associated pulmonary arterial hypertension treated with bosentan as first-line monotherapy followed or not by the addition of prostanoids or sildenafil. Rheumatology 2010; 49: 490-500.

30 D’Alonzo GE, Barst RJ, Ayres SM, et al. Survival in patients with primary pulmonary hypertension. Results from a national prospective registry. Ann Intern Med 1991; 115: 343-349.

31 Saggar R, Sitbon O. Hemodynamics in pulmonary arterial hypertension: current and future perspectives. Am J Cardiol 2012; 110: S9-S15.

32 Sitbon $\mathrm{O}$, Humbert $\mathrm{M}$, Nunes $\mathrm{H}$, et al. Long-term intravenous epoprostenol infusion in primary pulmonary hypertension: prognostic factors and survival. J Am Coll Cardiol 2002; 40: 780-788.

33 Avouac J, Kowal-Bielecka O, Pittrow D, et al. Validation of the 6 min walk test according to the OMERACT filter: a systematic literature review by the EPOSS-OMERACT group. Ann Rheum Dis 2010; 69: 1360-1363.

34 Humbert M, Singh M, Furst DE, et al. Pulmonary hypertension related to systemic sclerosis: points to consider for clinical trials. Rheumatology 2017; 56: v33-v37.

35 Sanges S, Giovannelli J, Sobanski V, et al. Factors associated with the 6-minute walk distance in patients with systemic sclerosis. Arthritis Res Ther 2017; 19: 279.

36 Gadre A, Ghattas C, Han X, et al. Six-minute walk test as a predictor of diagnosis, disease severity, and clinical outcomes in scleroderma-associated pulmonary hypertension: the DIBOSA study. Lung 2017; 195: 529-536.

37 Kylhammar D, Kjellström B, Hjalmarsson C, et al. A comprehensive risk stratification at early follow-up determines prognosis in pulmonary arterial hypertension. Eur Heart J 2017; doi: 10.1093/eurheartj/ehx257.

38 Hoeper MM, Kramer T, Pan Z, et al. Mortality in pulmonary arterial hypertension: prediction by the 2015 European pulmonary hypertension guidelines risk stratification model. Eur Respir J 2017; 50: 1700740.

39 Hoeper MM, Pittrow D, Opitz C, et al. Risk assessment in pulmonary arterial hypertension. Eur Respir J 2018; 51: 1702606 .

40 Condliffe R, Kiely DG, Peacock AJ, et al. Connective tissue disease-associated pulmonary arterial hypertension in the modern treatment era. Am J Respir Crit Care Med 2009; 179: 151-157.

41 Launay D, Montani D, Hassoun PM, et al. Clinical phenotypes and survival of pre-capillary pulmonary hypertension in systemic sclerosis. PLoS One 2018; 13: e0197112. 